\title{
A Case of Nivolumab-Associated Oral Lichenoid Lesions ${ }^{+}$
}

\author{
Andrea Spinelli *, Davide Bartolomeo Gissi, Roberto Rossi and Andrea Gabusi \\ Department of Biomedical and Neuromotor Sciences, Section of Oral Sciences, University of Bologna, 40125 \\ Bologna, Italy; davide.gissi@unibo.it (D.B.G.); roberto.rossi30@studio.unibo.it (R.R.); \\ andrea.gabusi3@unibo.it (A.G.) \\ * Correspondence: andrea.spinelli648@gmail.com; Tel.: +51-2088123 \\ + Presented at the XV National and III International Congress of the Italian Society of Oral Pathology and \\ Medicine (SIPMO), Bari, Italy, 17-19 October 2019.
}

Published: 12 December 2019

\section{Introduction}

Nivolumab is an anti-programmed cell death receptor-1 (PD-1) antibody recently approved for the treatment of melanoma, non-small-cell lung cancer, and several other solid tumours including head and neck cancers. By blocking the programmed cell death receptor-1 (PD-1), a checkpoint for the activation of the immune system, Nivolumab proved to enhance pre-existing immune responses inducing tumour recognition and attack. However, anti PD-1 blockage and related immune activation has also been related to adverse events including immunological skin, gastrointestinal, liver, endocrine system and other organ systems disorders. So far only few case reports have described lichenoid lesions triggered by nivolumab and restricted to oral cavity. In all, clinical appearance was that of multiple oral ulcers with histological features of lichenoid inflammation [1]. In this report we describe a case of reticular/erosive lichenoid lesions associated with nivolumab monotherapy.

\section{Case Report}

A 60 years old women was referred by her dentist for the appearance of burning oral lesions non responding to conventional treatments. At examination, reticular bilateral striae in a context of atrophic ulcerative erosion could be noted. The patient had received a diagnosis of thyroid aden ocarcinoma in 2017 and had been treated with nivolumab monotherapy ever since. In 2019 she switched from a $240 \mathrm{mg} / 2$ weeks regimen to a $480 \mathrm{mg}$ monthly administration. 2 months after the change in the therapeutic regimen lesions appeared. The patient was also taking ranipril for blood pressure control. An incisional biopsy was taken revealing a band-like infiltrate mainly composed of lymphocytes with evidence of basal membrane aggression. Direct immunofluorescence was negative. Given the close change in cancer therapy and in absence of other putative factors, a diagnosis of lichenoid lesions associated with anti-PD-1 antibody inhibitors was made. In accordance with her oncologist, the patient preferred not to suspend nor change nivolumab therapy giving priority to tumour response. For the same reason the patient preferred to delay therapy with local/systemic steroid as symptoms did not impact significantly on her quality of life. She is currently on regular follow up.

\section{Conclusions}

Lichenoid lesions associated with anti-PD-1 antibody inhibitors may manifest not solely as oral ulcerative lesions but may adopt different clinical variants being thus undistiguishable from oral lichen planus. Given the recent approval of anti-programmed cell death receptor-1 (PD-1) antibodies 
for many solid tumors the use of anti-PD-1 antibody inhibitors is expected to grow. Consequently, despite only few cases of oral lichenoid adverse reactions have been described and yet data regarding their clinical management is still limited, dentists should be informed of their existence in order to avoid delays in diagnosis and treatment. It is still unclear if concurrent medications may have a role in the pathogenesis.

Conflicts of Interest: The authors declare no conflict of interest.

\section{Reference}

1. Obara, K.; Masuzawa, M.; Amoh, Y. Oral lichenoid reaction showing multiple ulcers associated with antiprogrammed death cell receptor-1 treatment: A report of two cases and published work review. J. Dermatol. 2018, 45, 587-591, doi:10.1111/1346-8138.14205.

(C) 2019 by the authors. Licensee MDPI, Basel, Switzerland. This article is an open access article distributed under the terms and conditions of the Creative Commons Attribution (CC BY) license (http://creativecommons.org/licenses/by/4.0/). 